This item was submitted to Loughborough's Research Repository by the author.

Items in Figshare are protected by copyright, with all rights reserved, unless otherwise indicated.

\title{
Experimental measurement of local burning velocity within a rotating flow
}

PLEASE CITE THE PUBLISHED VERSION

http://dx.doi.org/10.1007/s10494-011-9331-6

\section{PUBLISHER}

(C) Springer Science+Business Media B.V.

\section{VERSION}

AM (Accepted Manuscript)

\section{LICENCE}

CC BY-NC-ND 4.0

\section{REPOSITORY RECORD}

Long, Edward J., and Graham K. Hargrave. 2019. "Experimental Measurement of Local Burning Velocity Within a Rotating Flow". figshare. https://hdl.handle.net/2134/13441. 
This item was submitted to Loughborough's Institutional Repository (https://dspace.lboro.ac.uk/) by the author and is made available under the following Creative Commons Licence conditions.

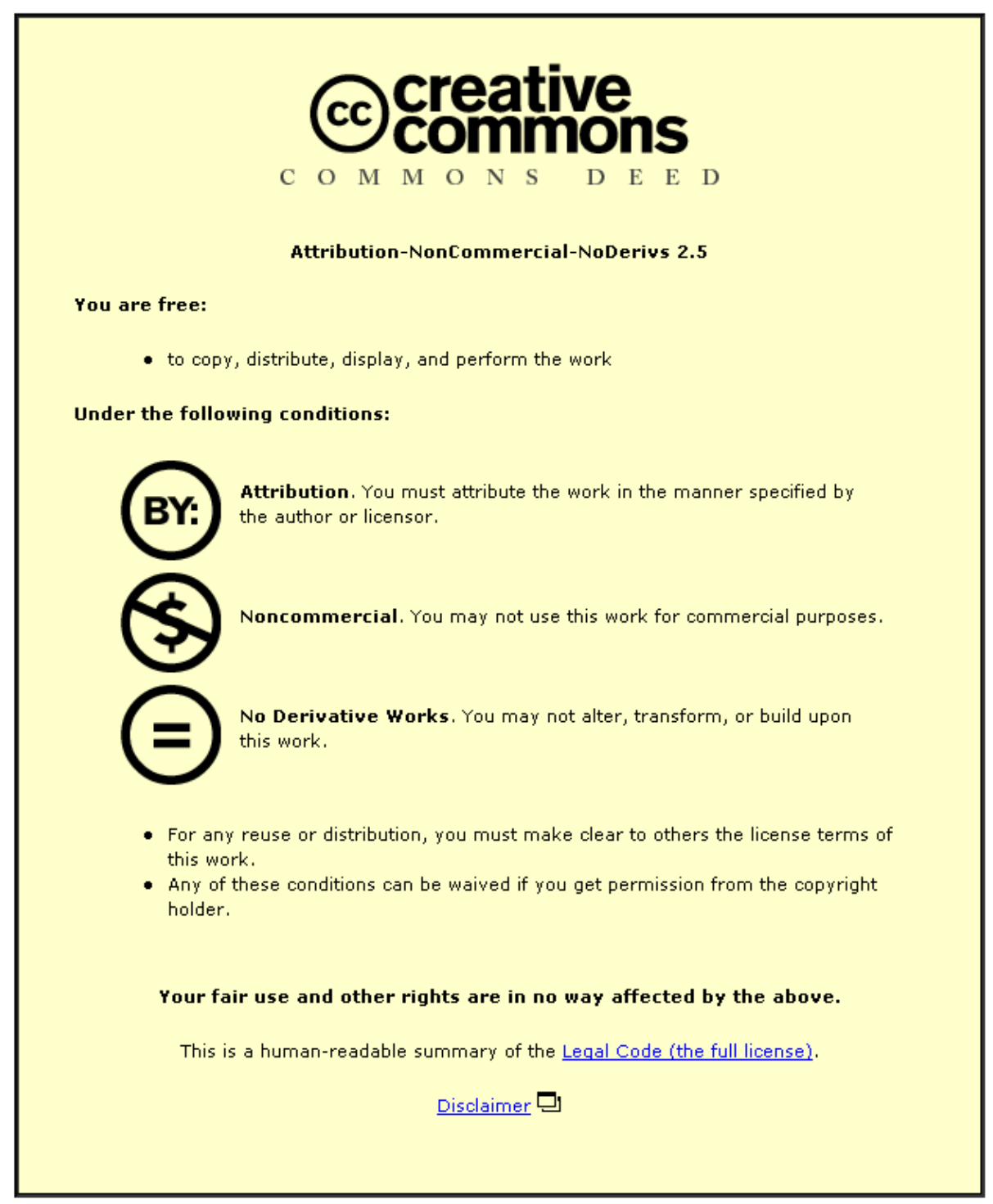

For the full text of this licence, please go to: http://creativecommons.org/licenses/by-nc-nd/2.5/ 


\title{
Experimental measurement of local burning velocity within a rotating flow
}

\author{
E. J. Long and G. K. Hargrave
}

Wolfson School of Mechanical and Manufacturing Engineering, Loughborough University, England

Tel: (+44)1509 227703

Fax: (+44)1509 227704

E.J.Long@lboro.ac.uk

Keywords: Burning velocity, PIV, combustion, vortex, local flame displacement

\begin{abstract}
The work presented in this paper details the implementation of a new technique for the measurement of local burning velocity using asynchronous particle image velocimetry. This technique uses the local flow velocity ahead of the flame front to measure the movement of the flame by the surrounding fluid. This information is then used to quantify the local burning velocity by taking into account the translation of the flame via convection. In this paper the developed technique is used to study the interaction between a flame front and a single toroidal vortex for the case of premixed stoichiometric methane and air combustion. This data is then used to assess the impact of vortex structure on flame propagation rates. The burning velocity data demonstrates that there is a significant enhancement to the rate of flame propagation where the flame directly interacts with the rotating vortex. The increases found were significantly higher than expected but are supported by burning velocities [22-24] found in turbulent flames of the same mixture composition. Away from this interaction with the main vortex core, the flame exhibits propagation rates around the value recorded in literature for unperturbed laminar combustion [1821].
\end{abstract}

\section{Introduction}

Turbulent burning velocity is an important parameter in the study of premixed combustion. Numerous studies, both experimental and numerical, have shown that a flames burning velocity is sensitive to local conditions such as strain [1-2] and curvature [3-6]. The degree of sensitivity in turn is governed by the nature of the reactants involved which define aspects such as thermo-diffusive instability and preferential diffusion [7]. Quantification of the turbulent burning velocity is not only utilised within the modelling of combustion events, such as in the level-set equation of direct numerical simulation (DNS) or the sub-grid scale models of large eddy simulation (LES), it is also a key parameter within the validation of these models [8]. As a result, experimental measurement of this variable is essential for the ongoing development of our understanding and simulation of combustion events. There are three common definitions of turbulent burning velocity: 
- Global consumption speed $\left(\mathrm{S}_{\mathrm{T}, \mathrm{GC}}\right)$

- Local consumption speed $\left(\mathrm{S}_{\mathrm{T}, \mathrm{LC}}\right)$

- Local displacement speed $\left(\mathrm{S}_{\mathrm{T}, \mathrm{LD}}\right)$

As argued in a review paper by Driscoll [8], all three of these approaches is a valid way to compare simulation to experiment, however it should be noted that for any specific flame these three quantities are not equal. An example of this is the work produced by Shepherd and Cheng [9], which demonstrated that for a given flame, local displacement speeds were consistently 2-3 times higher than the recorded local consumption speeds. Due to this inequality, the values produced using these different approaches cannot be directly compared to each other without first assessing the basis of their measurement. The current work is focused upon the measurement of local changes in flame propagation and so the use of global consumption speed is not valid, leaving local consumption and displacement speed for consideration. Both of these measurements are dependant on accurate optical measurement of flame progression; consumption speed requires assessment of both volumetric reaction rate and flame surface density, while displacement speed requires assessment of the local flow velocity and flame front movement. Local consumption speed is suitable for the measurement of highly wrinkled flame structures found within turbulent flows due to its volumetric approach. For this work, however, a modification of the usual displacement speed measurement is used to assess local flame burning velocity due to the relatively simple flame profile examined.

The use of displacement speed is well established for simple laminar flames propagating through unperturbed flow conditions; its use being applicable to the thin, slowly propagating flames whose development time scale is short in comparison to the time and length scales of the flow [10]. However, the local measurement of flame displacement speed in a truly turbulent field is a significantly more difficult challenge due to the reduced time scales and complex three dimensional flow. Despite this difficulty, it is still of interest to investigate how flame speed locally reacts to flow structures typically found within turbulent fields. In order to address this challenge, the work presented in this paper investigates the case of a laminar flame front interacting with a single toroidal vortex generated ahead of the flame front The use of this approach creates controlled highly repeatable flame-flow interactions that produce curvature and strain effects typically found within genuinely turbulent fields. Examples of three such interactions are shown in Figure 1, which displays images extracted from high speed laser sheet flow visualisation (HSLSFV) sequences of three different combustion events of premixed stoichiometric methane and air. The experimental test rig used to generate these interactions is described in section 2.1 of this paper. The vortices produced have the same core diameters $(\sim 6 \mathrm{~mm})$, but different rotational velocities, and it is clear from these images that there are significant differences in the flame-vortex interactions that develop; the apparent consumption rate of the main vortex core rapidly increasing with the rise in vortex rotation speed. 


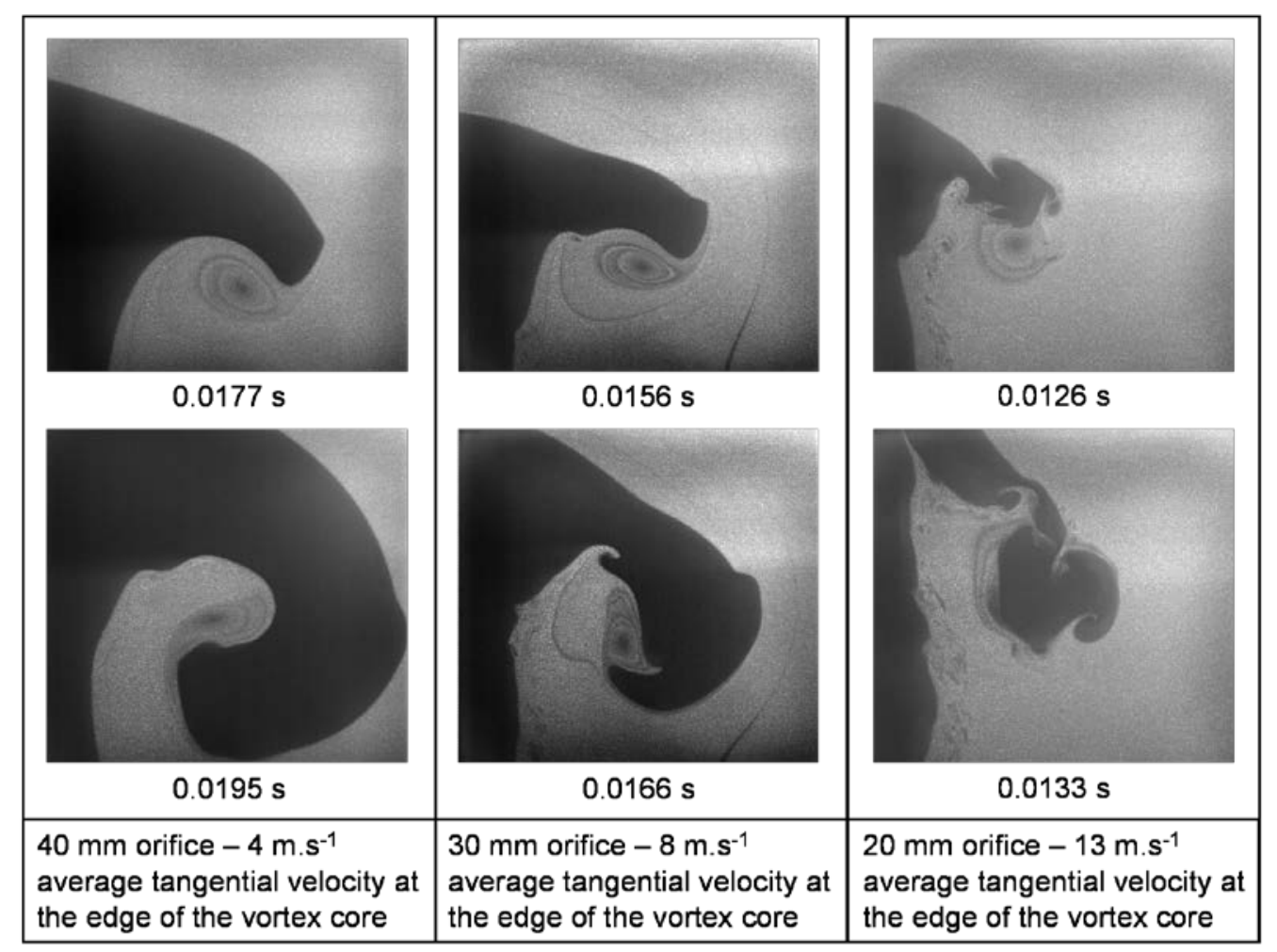

Fig. 1 Flame vortex interactions under different rotational velocities. Captions indicate time from ignition

To enable further understanding of the underlying reasons of this change in behaviour, the work in this paper presents an experimental investigation into an analysis technique aimed at quantifying the local burning velocity based on displacement speed. The flame-vortex interaction produced by the $30 \mathrm{~mm}$ orifice case shown in figure 1 is used as the basis of this investigation.

For this study, the combustion of premixed stoichiometric methane and air is experimentally measured, with initial conditions of atmospheric pressure and temperature.

\subsection{Local displacement speed measurement}

The standard definition of local flame displacement speed is given in equation $1[8]$.

$$
\mathbf{u}_{t}=\left[\mathbf{V}_{F} \cdot \mathbf{n}\right]-\left[\mathbf{V}_{G} \cdot \mathbf{n}\right]
$$

Examples of this approach include the work carried out by Renou et al. [11] and Sinibaldi et al. [12]. The basis of this work is that the local burning velocity $\left(\mathbf{u}_{\mathrm{t}}\right)$ is measured as being the difference between the local flame velocity $\left(\mathbf{V}_{\mathrm{F}}\right)$, measured normal to the flame front (n), and the local flow velocity in the same direction $\left(\mathbf{V}_{\mathrm{G}}\right)$.

This approach to measuring the rate of flame propagation can provide poignant information as it takes into account the high levels of flame displacement caused by the fluid flow. As a result, meaningful information on flame-flow interaction can be determined. However, this approach encounters problems when trying to deal with a reactant flow that contains a strong tangential component relative to the flame front such as that found eddy structures. In these situations, 
where a strong tangential flow is comparable to or greater than the burning velocity, a point on the flame front can be moved or convected a considerable distance away from a direction normal to the flame front, as detailed in figure 2.

Fig 2 Schematic of flame displacement in a direction normal to the flame front. Flame A represents the position at time t, Flame $\mathrm{B}$ represents the position at time $\mathrm{t}+\mathrm{dt}$.

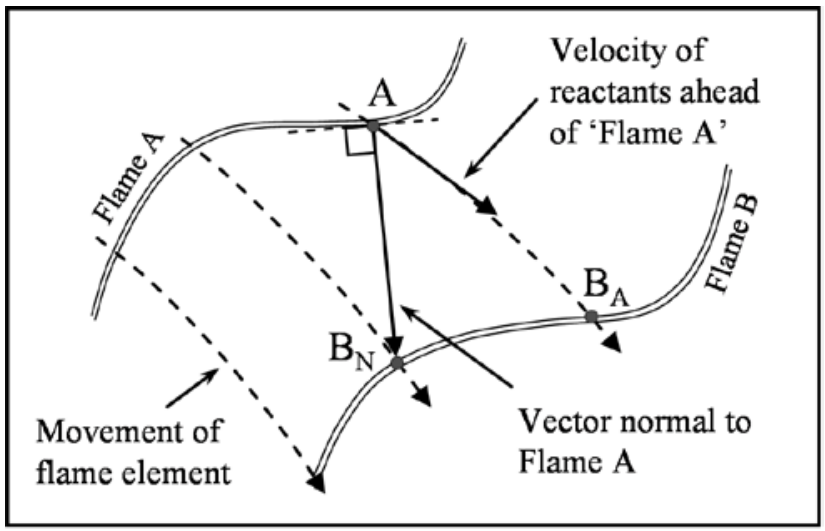

This translation of the flame means that if only a movement normal to the flow velocity is considered, two very different sections of the flame are related by this approach, resulting in a false burning velocity measurement. It is apparent that this technique of measuring burning velocity is not able to adequately separate the two factors of flame advection and flame propagation for the case of highly rotational flow. Therefore, if the true response of a flame front to local flow conditions is to be established, a different technique is required that can cope with flows that have a strong tangential flow relative to the flame front

To meet this need, this work implements a different approach to measuring local displacement speed, based on optical measurement, to calculate local burning velocity. The foundation of this approach is the use velocity data, acquired using asynchronous particle image velocimetry (APIV), to predict the convection of the flame that would occur over a small period of time, creating a 'virtual' flame front (see figure 3). This virtual flame is then compared with the actual flame translation during this same time interval. The difference between the actual and virtual flame fronts provides the local flame displacement due to propagation, and thus can be used to calculate burning velocity.

Fig. 3 Burning Velocity Calculation using Flame Advection. Flame A represents the position at time $\mathrm{t}$, Flame $\mathrm{B}$ represents the position at time $\mathrm{t}+\mathrm{dt}$.

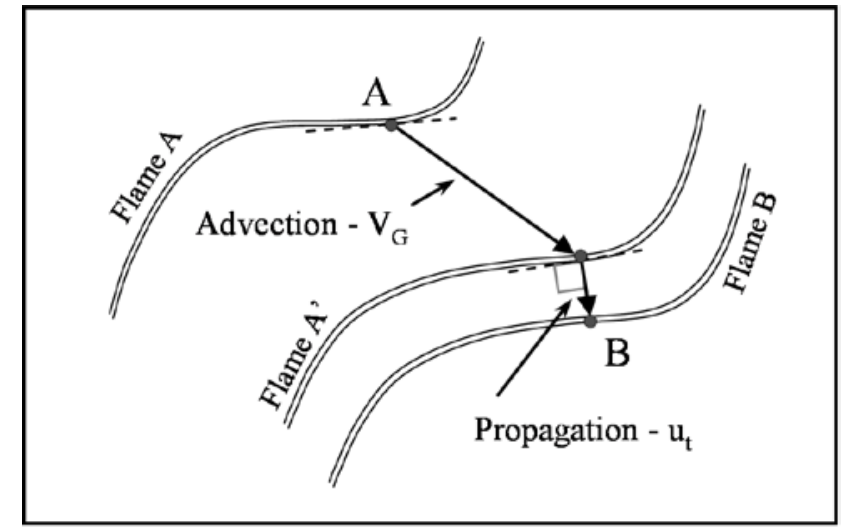

Using the velocity of the surrounding charge to convect the flame front to a theoretical position, prior to the measurement of burning velocity, enables its impact on the flame movement to be included regardless of its magnitude and direction. The flame is still considered to propagate in a direction normal to its surface, a view that is supported by the results shown in many studies of flame response to curvature and stretch $[1,5,6,8,9,11]$. More information regarding the 
experimental technique employed is included in section 2. This approach, previously presented by the authors in 2008 [13], has subsequently been employed to study flame propagation under low turbulence conditions by Hartung et al.[14]. In their work stereoscopic particle image velocimetry was used in conjunction with $\mathrm{OH}$ planer laser induced fluorescence (OH-PLIF) to track the movement of the flow and flame respectively. Turbulent structures within a flame produced by an acoustically forced bluff body burner were examined in order to determine local displacement speed. Although stereoscopic PIV was employed, the third component flame motion was not captured by the OH-PLIF images resulting in a two dimensional measurement of a three dimensional event. Only an estimation of the third component was incorporated. In the work presented in this paper, this problem of the third component is mitigated by using an axisymmetrical flamevortex interaction. With this configuration, when taking a cross section through the central axis of the toroid, out of plane motion minimal, its effect predictable due to symmetry of the interaction.

\section{Experimental arrangement}

\subsection{Combustion bomb setup}

In order to generate a controllable and repeatable interaction between a rotating flow structure and a propagating flame front, a twin chamber combustion bomb was designed and manufactured, a schematic of which is shown in figure 3. This rig was operated by igniting a premixed charge of methane and air inside a small cylindrical pre-chamber linked to a much larger main combustion chamber via an orifice. The fuel-air mixture was prepared prior to introduction into the combustion chamber, using mass flow controllers to meter the reactants. This mixture was then allowed to purge through the combustion chambers for 2 minutes to ensure a homogeneous charge was achieved throughout. After this period, the solenoid valves at the inlet and exit were closed and the movement of the contained mixture allowed to settle for a further minute before ignition.

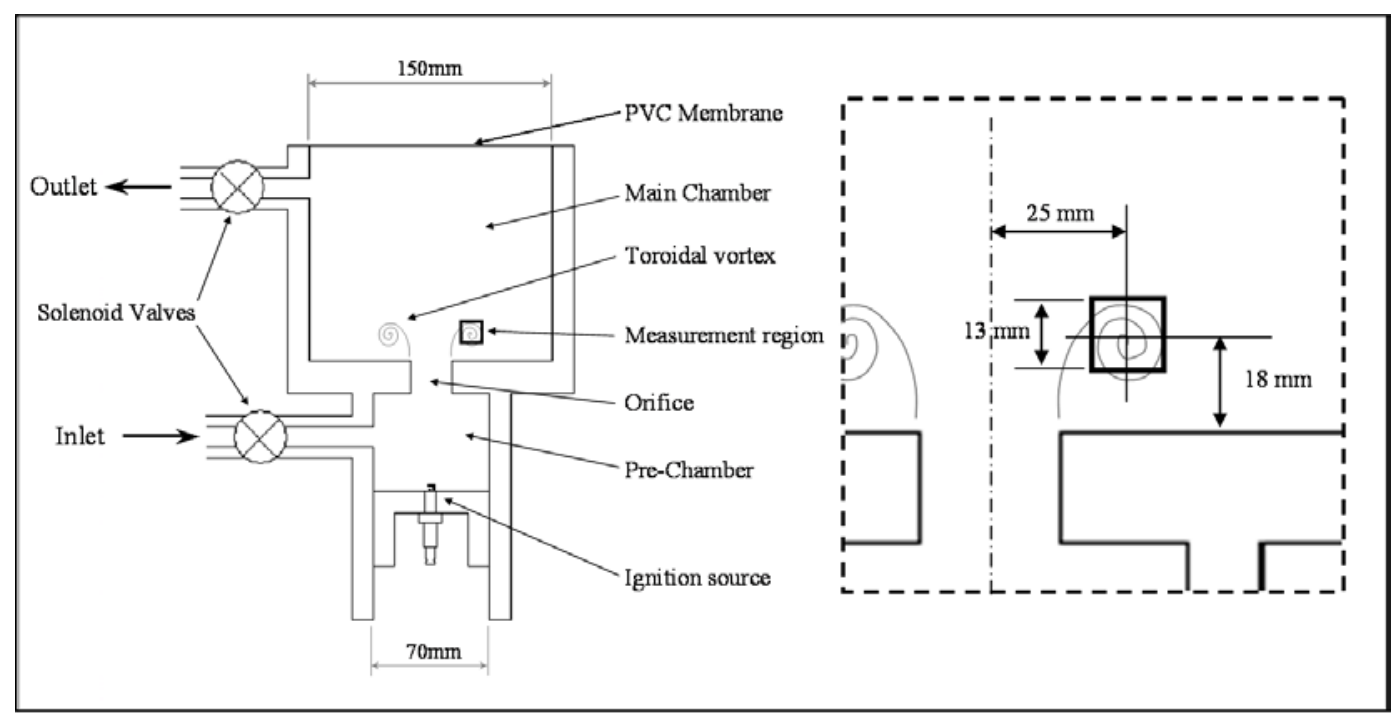

Fig. 4 Schematic of the combustion bomb and an image of the combustion event 
After ignition, a toroidal vortex structure was formed within the main combustion chamber by the action of the flame-front propagating through the prechamber and pushing unburned reactants ahead of it through the orifice. This charge movement interacting with the static charge within the main chamber created a shear layer at the orifice exit, which in turn created a vortex. As the flame continued to propagate through the charge, it interacted with this vortex structure, distorting the flame and altering its burning velocity. The combustion chamber was sealed using a PVC membrane which ruptures soon after ignition allowing the chambers to vent, preventing excessive pressure build up. For the work presented in this paper a $30 \mathrm{~mm}$ diameter orifice was used that had a restriction length of 25mm and 90 degree corners at both the inlet and exit faces. The subsequent vortex produced from this arrangement had a close approximation to a Rankine vortex; i.e. a forced rotational vortex core surrounded by an irrotational couette type flow structure. The diameter of the core produced was approximately $6 \mathrm{~mm}$ in diameter. The resultant flame-vortex was highly repeatable in nature, enabling results from different events to be compared.

\subsection{Optical Diagnostics}

In order to quantify the movement of both the flame and the surrounding flow a modification to the asynchronous PIV (APIV) technique employed by Jarvis et al. [15] was employed (see figure 5). Particles of $1 \mu \mathrm{m}$ olive oil were used as the seeding material so that, not only could the velocity of the unburned gas be recorded, but also the position of the flame edge could be identified by its consumption.

For the APIV, two completely independent PIV systems, operated in single shot mode, were used to capture the motion of the flame and reactant flow in the same region; the only link between these two systems being a multiple signal generator that provided control of the temporal delay between ignition and the image acquisition. In order that the two PIV systems did not interfere with each other, the light produced from each double pulse laser was set to a different polarisation, 90 degrees apart. With polarisation filters in front of the camera lenses rotated to the correct angle, in conjunction with a polarising beam splitter, each camera only saw the scattered light from its matched laser. With this arrangement, zero cross-talk between the two-systems was measured. Using this approach, two sequential vector fields can be recorded with any time separation without one set interfering with the other. As a result of this setup, events which occur over very short time intervals can be investigated without issues such as laser power or camera resolution limiting the results.

Fig 5 Schematic of asynchronous PIV layout

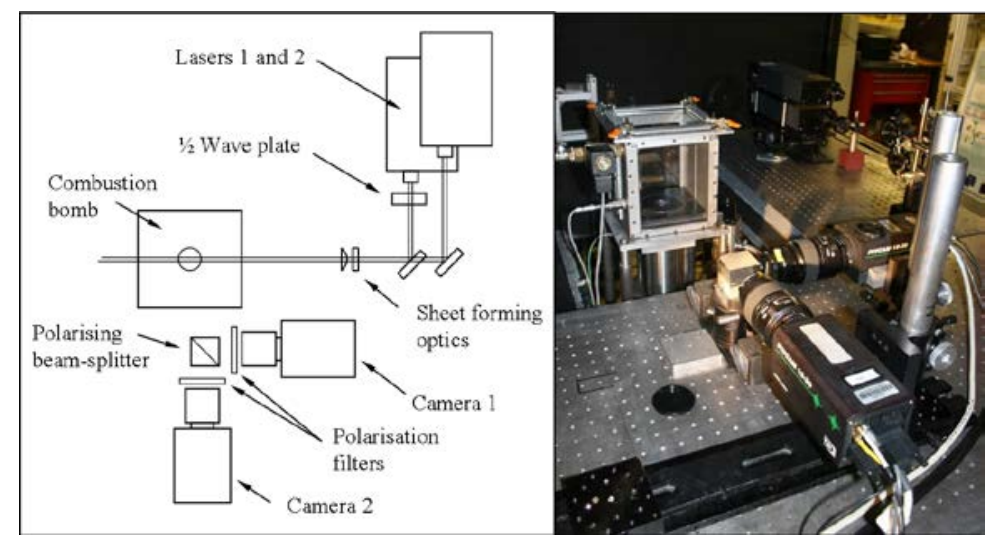


Using APIV sequentially introduces significant complexity when trying to measure burning velocity in the method described in section 2 . The reason for this difficulty is that the image areas of the two systems have to be perfectly aligned in order to make an accurate measurement of flame displacement; a situation that is hard to achieve. Modifying the timing of the two APIV sets so that they start at the same time but one set measures the flow velocity and one set measures the flame displacement, mitigates this alignment issue resulting in a more accurate flame speed measurement. A timing diagram of this setup is shown in figure 6; set 1 records the images used for the flow velocity measurement, set 2 records the information for the flame displacement measurement.

Fig 6 APIV timing

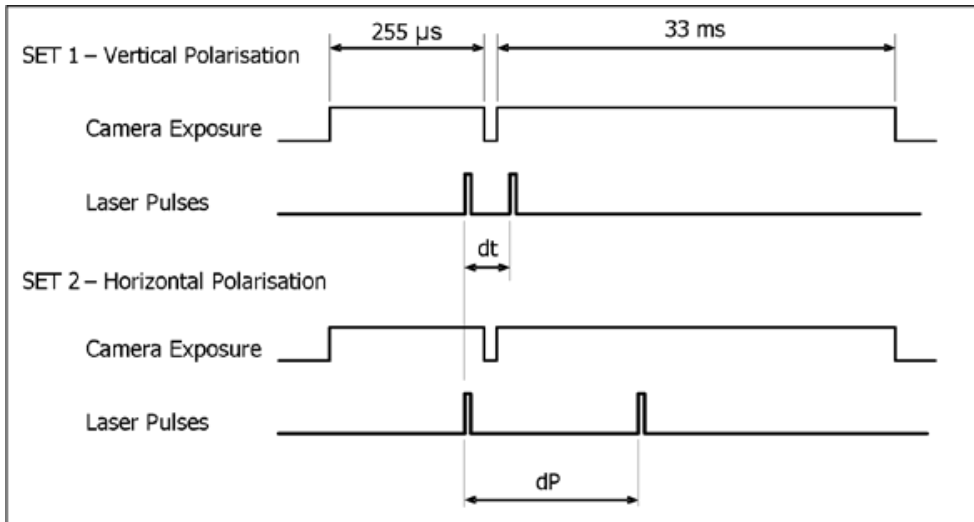

The accuracy of the final burning velocity measurement is highly dependent on the temporal and spatial resolution achieved in the image acquisition. For this reason the area of the images used is kept to a minimum with respect to the interaction being examined; in this case a region 13 by $13 \mathrm{~mm}$ was examined. Details of the image acquisition settings are given in table 1.

Table 1: APIV image acquisition settings

\begin{tabular}{|l||c|}
\hline Time between images for set $1(\mathrm{dt})$ & $8 \mu \mathrm{s}$ \\
\hline Time between images for set 2(dP) & $80 \mu \mathrm{s}$ \\
\hline Image region size & 13 by $13 \mathrm{~mm}$ \\
\hline Image Resolution (pixels) & 1000 by 1016 \\
\hline Lens & $105 \mathrm{~mm}$ macro \\
\hline Focal length over aperture ratio (F\#) & 8 \\
\hline Diffraction limited particle image size & 2.8 pixels \\
\hline Distance from image centre to orifice centre & $25 \mathrm{~mm}$ \\
\hline Distance from image centre to chamber floor & $18 \mathrm{~mm}$ \\
\hline
\end{tabular}

With regard to the time between images for sets 1 and 2, these values were kept to a minimum relative to the spatial resolution of the images. In particular, set 2 was used with a dP time of $80 \mu \mathrm{s}$ in order to provide sufficient spatial resolution of the flame propagation after the convection process. If this value is too small, relative to the spatial resolution with which the flame edge can be established, significant error occurs. 


\subsection{Image Analysis and adaptive grid cross-correlation}

In order to analyse the images and return a measurement of local burning velocity, a bespoke computational program was developed. The produced program operated directly on the digital images taken from the APIV technique and incorporated all aspects of the flame and flow velocity measurement. The first stage of the program identified the location of the flame edge, tracking its progression from one image to the next. The location of the flame edge is defined in this work by the disappearance of the oil particles as they evaporate in the preheat zone of the flame front. In their work on partially premixed methane-air combustion, Heeger et al. [16] reported an offset of between 0.5 and $1 \mathrm{~mm}$ between the leading flame edge defined using OH-PLIF and the evaporation of $1 \mu \mathrm{m}$ olive oil particles. The flame edge is identified from the images by initially enhancing the low level light present in the images. These low intensity particles help to distinguish the difference between the burned and unburned regions but contribute little to the signal utilised by the velocity measurement process. The flame edge is then identified from the sharp gradient in light intensity present at the burned/unburned gas interface, see figure 7.

Fig 7 Flame front position taken from APIV images

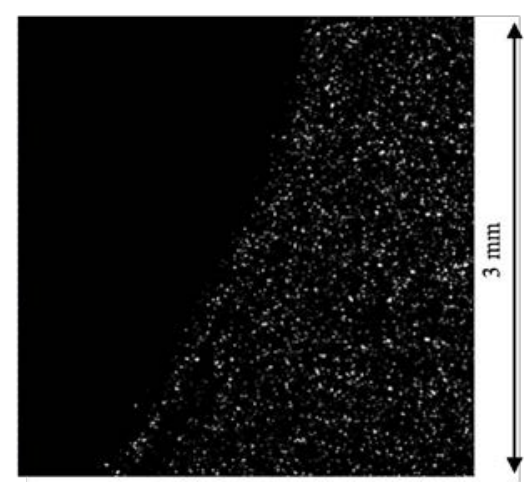

a) Raw PIV image

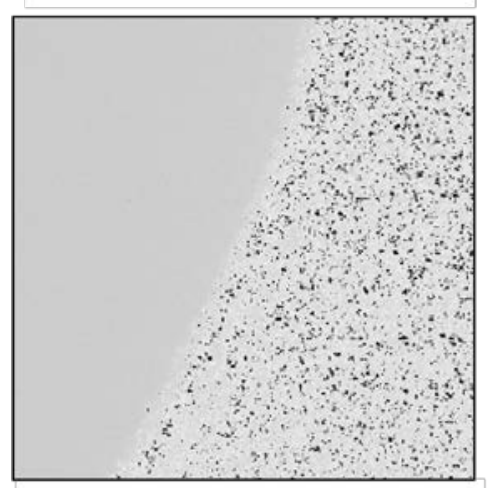

b) Intensity plot of PIV image (0-255 scale)

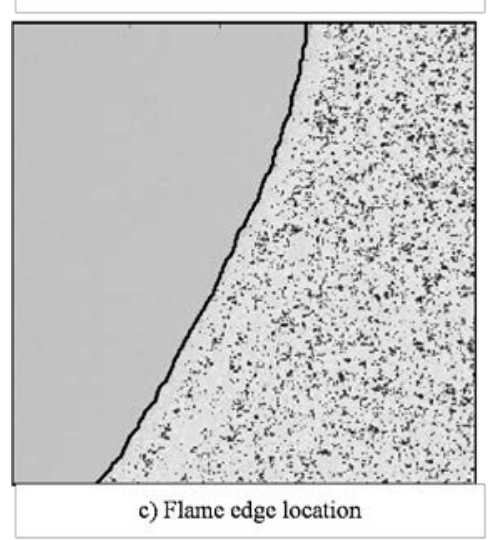


Having traced the first flame position, the software measures the flow velocity around the flame edge by adapting the position of the measurement location grid to match the recorded flame profile. An example of a typical interrogation region is given in figure 8 . In order to achieve high spatial resolution and accurate velocity data, an interrogation region size of 25 by 25 pixels was utilised for the first image (frame A, set 1); with a larger interrogation region of 35 by 35 pixels used the corresponding in the second image (frame $\mathrm{B}$, set 1 ). This corresponds to a spatial size of $0.325 \mathrm{~mm}$ square for the cross correlation process. A point on the flame edge is taken as the centre of the interrogation region, so that the signal that contributes to the velocity measurement is naturally located along a strip close to the flame, this reduces the impact of the flow velocity that is further out from the flame. Using solid seeding particles such as alumina could enable velocities closer to the inner reaction zone to be obtained, however, the large thermal gradients within the preheat and inner reaction zones result in a significant change in the refractive index of through which the particles are imaged, distorting their relative position. This distortion could provide significant error in the velocities measured. Normalised signal strength (NSS) crosscorrelation [17] was used to analyse the particle displacement in each interrogation region. NSS holds distinct advantages over standard FFT crosscorrelation due to its inherently low noise floor in the presence of signal level changes; a feature which is highly relevant for this application. This low noise floor results in a reduced RMS error and thus more accurate velocity data. Additionally, the low noise floor means that smaller interrogation regions can be utilised, reducing the impact of high velocity gradients as often found in rotating flows. The two images taken from the first PIV set (with an $8 \mu$ s pulse separation time) are then used with this grid to deduce the flow velocity convecting the flame. Using the flame edge profiles and the velocity measurements, the program calculates the local burning velocity using the technique described in section 1.1.

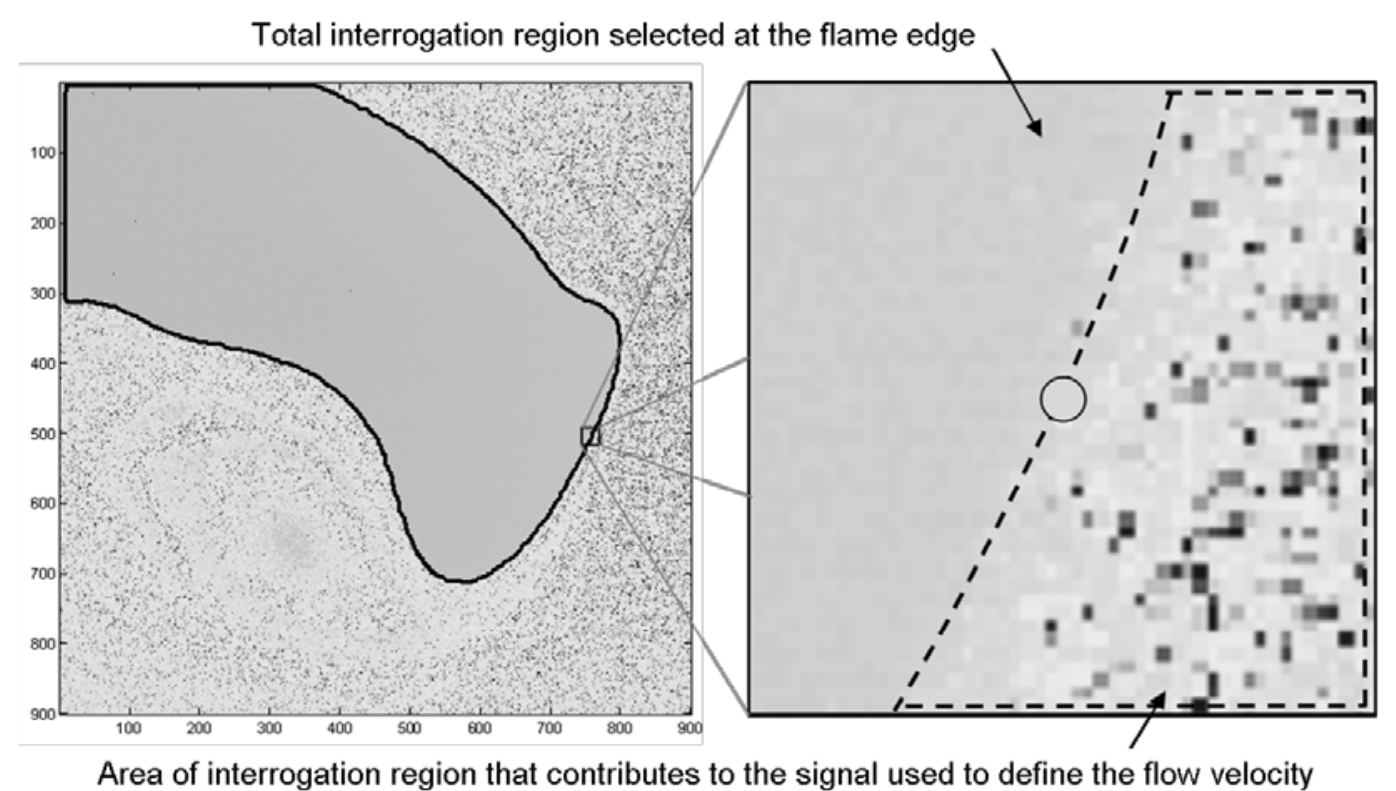

Fig 8 Interrogation region position

In order to track the flame edge with sufficiently high fidelity, as well as utilise small interrogation regions for the velocity measurement, the seeding density had to be set quite high. However, as $1 \mu \mathrm{m}$ droplets were used, the volume fraction of olive oil in the combustion bomb was low. The images indicate a 
higher spatial density of oil than reality due to particle image diffraction; the $1 \mu \mathrm{m}$ particles appear as $25-35 \mu \mathrm{m}$ particles in the image.

\section{Results}

Using the developed technique and analysis program, the effect of flow rotation on local burning velocity was examined using the combustion bomb described in section 3.2. Figure 9 contains an example of the raw images gathered using this technique and the subsequent velocity data calculated using commercial PIV analysis software. This velocity analysis was carried out using fast Fourier transform cross correlation, with a regularised grid of interrogation regions 32 by 32 pixels in size, overlapped by 50\%. Overlaid on the vector diagram are stream lines of the flow movement which have been included for clarity.

Fig 9
a) A raw
image of the
laser
illuminated
particles and
b) velocity
field of the
unburned
reactants
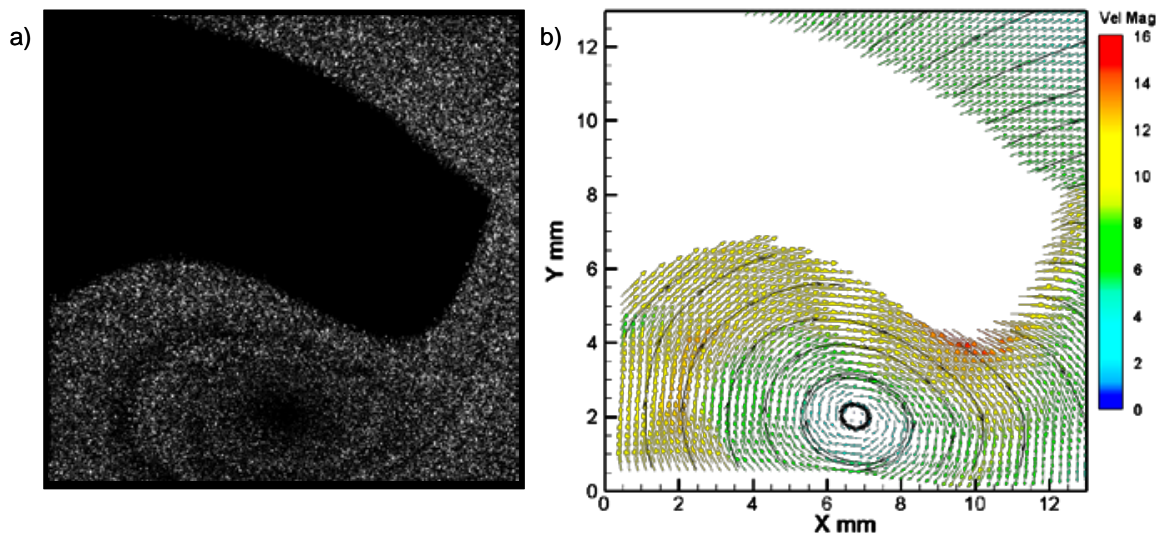

Figure 10a shows the resultant flame front locations extracted from the APIV images and the theoretical convected flame position. Figure 10b shows the resultant local burning velocity calculated from flame positions shown in 10a.

Fig 10

a) Extracted flame front locations and b) resultant local burning velocity profile
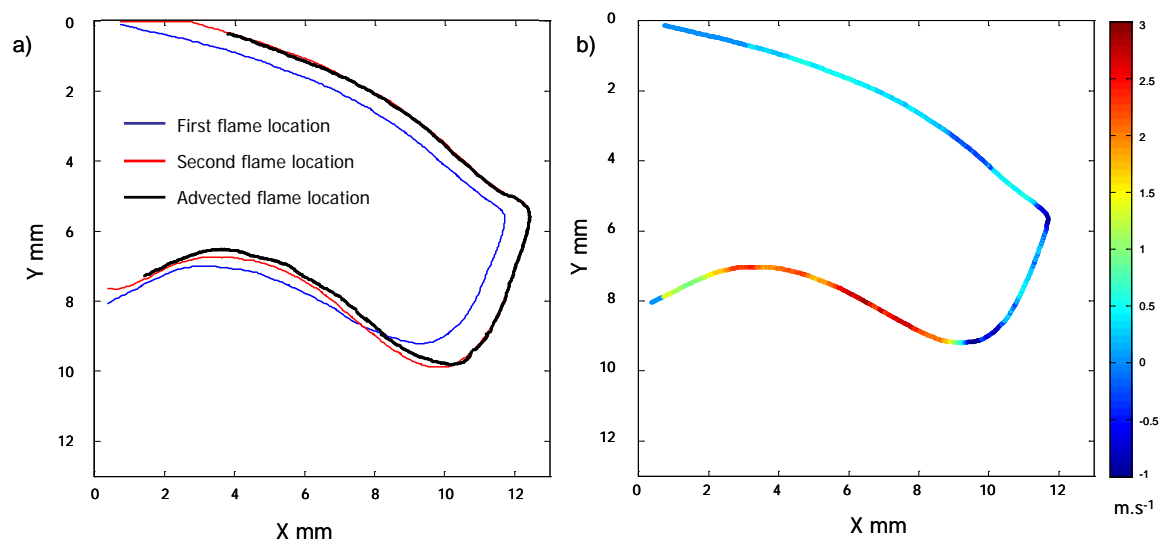

In figure 11 the full sequence of vector fields recorded of the flame interacting with the toroidal vortex is shown, included are streamlines of the flow movement for clarity. Overlaid on this plot are the burning velocity measurements. Due to the single shot nature of the experiment, each time step in the sequence was recorded from a different combustion event. Such am approach was made possible due to the high repeatability observed in the experiment. 


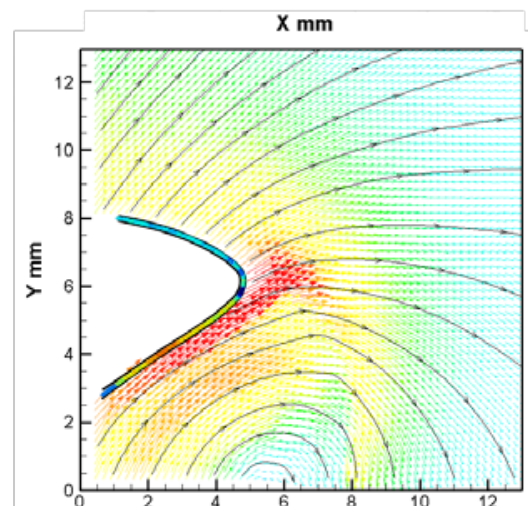

a) $15.25 \mathrm{~ms}$

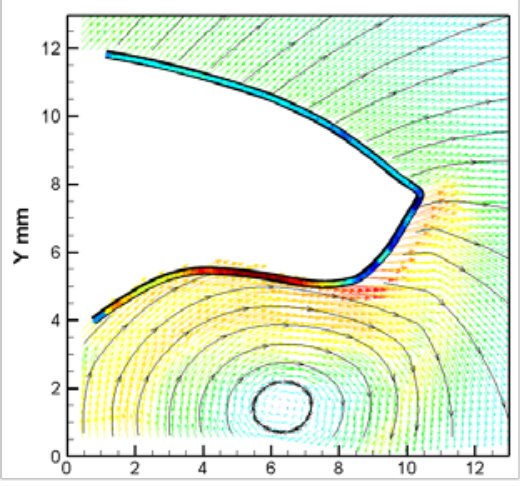

c) $15.75 \mathrm{~ms}$

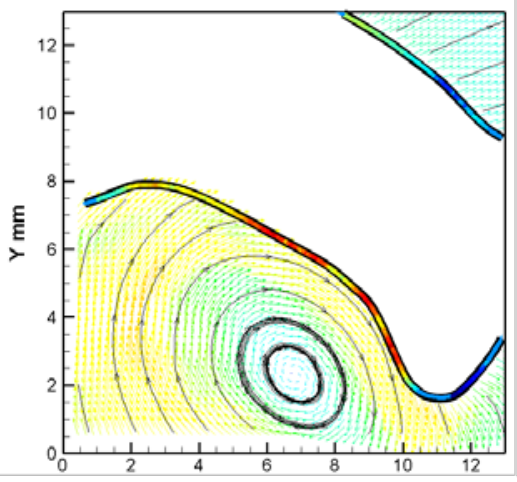

e) $16.25 \mathrm{~ms}$

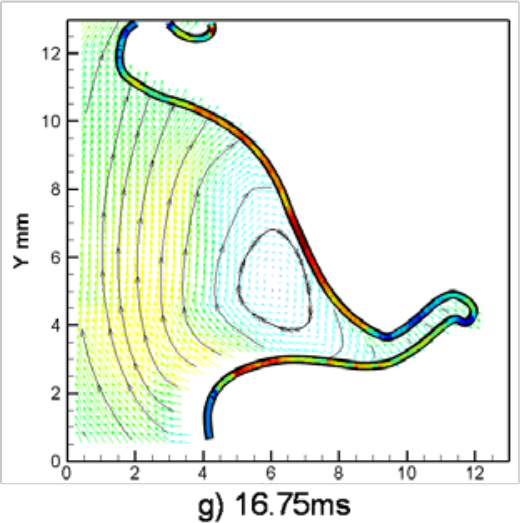

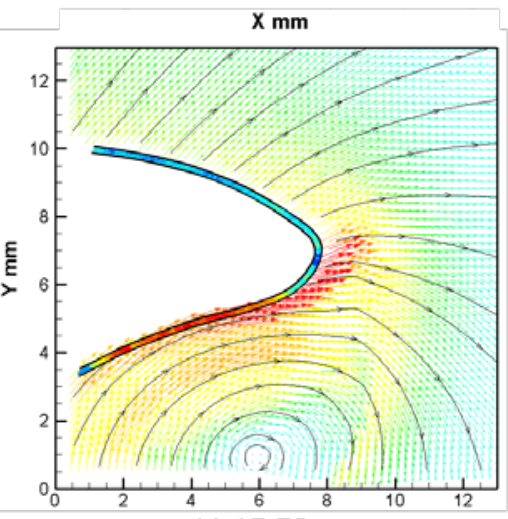

b) $15.50 \mathrm{~ms}$

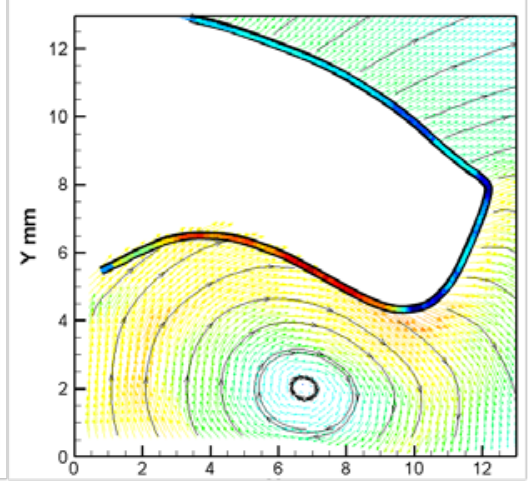

d) $16.00 \mathrm{~ms}$

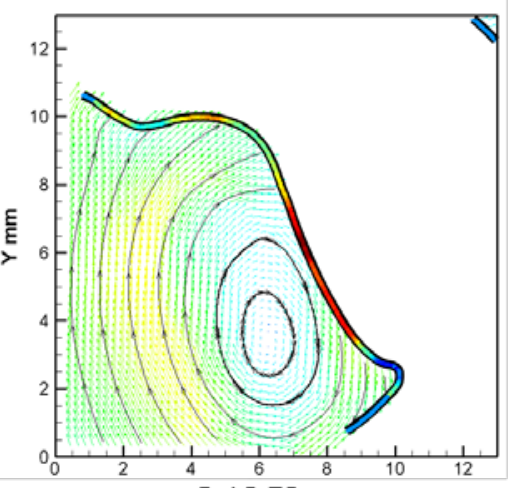

f) $16.50 \mathrm{~ms}$

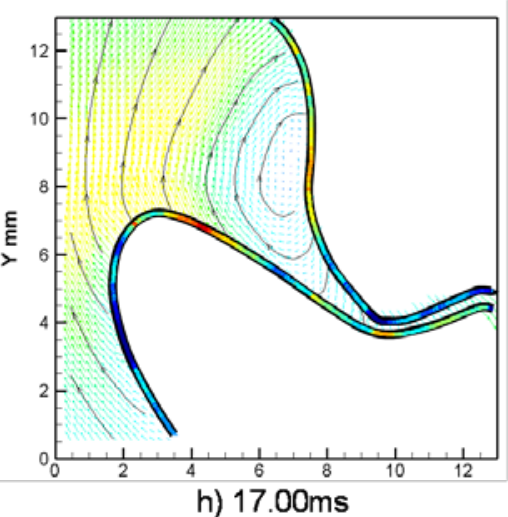

h) $17.00 \mathrm{~ms}$
Flow

velocity

m.s ${ }^{-1}$

16

14

12

10

8

6

4

2

0

Burning

velocity

m.s - $^{-1}$

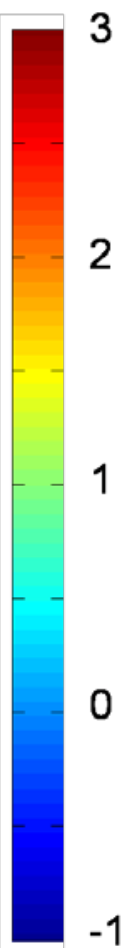

Fig 11: Velocity vector fields and burning velocity measurements of the interaction between a toroidal vortex structure and a propagating flame front. Times indicated are relative to ignition, streamlines are included for clarity. 
For the burning velocity measurement presented there are four potential sources of error: (i) the identification of the flame front position, (ii) the inaccuracy of the fluid velocity measurement, (iii) the inability of the process to fully account for flow curvature, and (iv) the lack of acceleration/deceleration information in the flow velocity measurement.

In terms of the flame position error, this is directly controlled by the ratio between the image area and the camera resolution. For the setup described in this paper, this results in an uncertainty of $\pm 6.5 \mu \mathrm{m}$ for each flame edge identified due to pixel locking. Leading on from this, the error in the cross-correlation of the interrogation regions has been shown to be under $2 \%$ for NSS. Combining these two sources of error results in an uncertainty of $\pm 0.48 \mathrm{~m} . \mathrm{s}^{-1}$ in the worst case (where the reactants are travelling at $16 \mathrm{~m} . \mathrm{s}^{-1}$ ). However, due to the lower flow velocities, the majority of the flame front will exhibit an uncertainty of less than $\pm 0.3 \mathrm{~m} . \mathrm{s}^{-1}$. The error associated with the flow characteristics of curvature and deceleration can be quantified via assessment of the velocity vector fields. This evaluation indicates that, in the worst case, there is a possible overestimation of up to $0.23 \mathrm{~m} . \mathrm{s}^{-1}$ at the tip of the flame, primarily due to deceleration, and up to 0.4 $\mathrm{m} . \mathrm{s}^{-1}$ along the edge of the vortex core due to curvature. Despite these high levels of error in comparison to the magnitude of the burning velocity, the overall trend of the data is unaffected.

\section{Discussion}

\subsection{Observations of the interaction}

The discussion of the recorded flame propagation will describe the flame/flow interaction processes defined by the development of the unburned gas velocity field and the local flame displacement speed shown in figure 11 . To aid the discussion, different regions of the interacting flame will be referred to according the labels given in figure 12 .

Fig 12. Flame sections

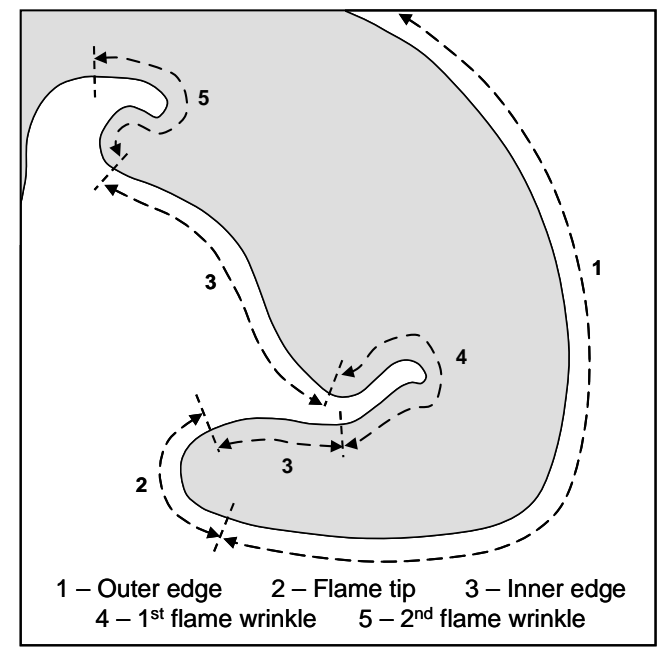

If the first four burning velocity data sets are considered (between $15.25 \mathrm{~ms}$ and $16.00 \mathrm{~ms}$ after ignition) an obvious distinction can be made between the section of the flame front in direct contact with the vortex (section 1) and the section along the outer edge, away from the rotation (section 3). In addition to 
these two sections, another region of flame front can also be identified from the results, the section close to the flame tip at the transition between the inner and outer edges of the flame (section 2).

The data recorded in section 1 of the flame demonstrates a relatively low rate of flame propagation, with average velocities of $0.39 \mathrm{~m} . \mathrm{s}^{-1}, 0.28 \mathrm{~m} . \mathrm{s}^{-1}, 0.37 \mathrm{~m} . \mathrm{s}^{-1}$ and $0.31 \mathrm{~m} . \mathrm{s}^{-1}$, for sets A, B, C and D respectively. Given the possible errors present within the measurements, these average results are in close approximation to the unperturbed laminar burning velocities presented in literature [18-21], indicating that this region of the flame does not experience any enhancement to the rate of propagation. The following section of the flame (section 2), identifiable by its close proximity to the flame tip, exhibits fluctuations in measured burning velocity, often producing negative values. These negative values are not a problem in themselves as it has been shown by the computational work of Gran et al. [4] that in regions of high positive curvature, as found in this part of the flame, negative burning velocities are possible. More discussion of the mechanisms supporting this are given in section 4.2. However, the degree of vacillation in the data indicates that limitations to the dynamic range of the displacement speed measurement technique used in this study generates error in the assessment of the flame-flow interaction within this region. The source of this possible error is the high flow velocities present at the flame tip with respect to the burning velocity. This disparity in the magnitude of the advection and propagation velocities means that the error contained within the PIV measurement of the local flow velocity results in a significant uncertainty in the calculated burning velocity. The final section of the flame front (section 3) directly interfaces with the vortex rotation and demonstrates a significant increase in burning velocity in comparison to the previous two. The results show an increase in the burning velocity as the flame starts to fully encounter the rotation, rising from a peak of $2 \mathrm{~m} . \mathrm{s}^{-1}$ at $15.75 \mathrm{~ms}$ after ignition to around $3 \mathrm{~m} . \mathrm{s}^{-1}$ by $15.75 \mathrm{~ms}$. The error analysis previously conducted indicates that the burning velocity measurement is subject to a possible overestimation of $0.23 \mathrm{~m} . \mathrm{s}^{-1}$ due to deceleration, and $0.45 \mathrm{~m} . \mathrm{s}^{-1}$ due to curvature. However, even in these worst case scenarios a combined reduction of $0.68 \mathrm{~m} . \mathrm{s}^{-1}$ to the values obtained for section 3 would not remove the fact that these results indicate that the burning velocity is significantly enhanced in this region of the flame. This variation in propagation rate corroborates with the observation of a bulge occurring in the flame front (Figure 11f and 11g) within section 3 as the flame front burns across the flow lines towards the centre of rotation. These values of burning velocity are higher than expected, more discussion of which is given in section 4.2

As the flame continues to develop in size and shape, section 1 of the flame edge becomes displaced outside of the image region (figure 11, f-h). In addition to this change, as part of the evolution of the flame front, two new regions of the flame become apparent. The first of these new developments is the formation of a wrinkle in the flame profile (section 4), positioned along the flame edge adjacent to the vortex. This flame wrinkle divides section 3 (the region directly interacting with the vortex) in two, each sub-section still being in contact with the vortex core. This wrinkle in the flame appears to develop due to the high propagation rate produced in section 3 . The formation of the bulge in this region of the flame means that part of the flame front burns across the flow lines of the vortex ahead of the rest of the flame. As this occurs, this small section of the flame then starts to interact with the fast moving flow present around the edge of the vortex core (figure 11e and 11f). The fast moving flow region translates this part of the flame 
more rapidly around the vortex, resulting in a fold forming in the previously smooth flame front. As the rest of the flame front continues to exhibit a higher burning velocity due to the vortex, this fold in the flame continues to develop, forming a wrinkle in which the trapped reactants are slowly consumed. The burning velocity measurements in section 4 of the flame show substantially lower values than section 3 which it divides. However, a high degree of oscillation starts to develop in the measurements around the cusp as the flame starts to encroach on this small volume of unburned reactants. This error in burning velocity is due to the small number of particles present within the trapped volume, reducing the signal available for accurate velocity measurement, this development can be seen by the occurrence of small peaks in the velocity data.

The other new section to appear in the burning velocity data is a region where the flame begins to wrinkle above the main vortex rotation (section 5). This wrinkle in the flame front is due to the flame front burning into the vortex at the same time as the fast moving flow as the edge of the vortex core pushes into the burned reactants. This interaction between the high velocity flow of the vortex and the encroaching burned reactants causes the flame to buckle and roll around the flow. The scale of this interaction is small in comparison to the image region used in this analysis, therefore no quantitative information can be extracted in terms of the burning velocity.

\subsection{Mechanisms of enhanced flame displacement speed}

The high burning velocities show in figure 11 represent a 4 to 8-fold increase from the unperturbed laminar burning velocities of between 0.35 and $0.38 \mathrm{~m} . \mathrm{s}^{-1}$ reported in literature [18-21]. This high multiple becomes reduced if the possible errors and overestimation discussed at the end of section 3 are taken into account, reducing the ratios of $\mathrm{S}_{\mathrm{T}, \mathrm{LD}} / \mathrm{S}_{\mathrm{L}, 0}$ to between 3 and 6 . However, even considering this reduction, the large increases in burning velocity are surprising considering the perturbed laminar nature of the flame and the stability of the reactants. Given the difficulty of measuring meaningful burning velocities from the type of flow under examination, there is little data from perturbed laminar flame experiments to support these findings. However, there is a wealth of information regarding the propagation of turbulent flames that provides useful reference. The basis of this comparison, as stated by Lipatnikov and Chomiak [7], is that a substantial role is played by thin laminar zones within turbulent flame propagation. Furthermore, all known concepts of predicting diffusivity effects on flame speed are based on perturbed laminar flames. There are numerous studies, both numerical and experimental, which have shown that the ratio between turbulent burning velocity and unperturbed laminar velocity $\left(\mathrm{S}_{\mathrm{T}} / \mathrm{S}_{\mathrm{LO}}\right)$ can be considerable for stoichiometric methane and air. For example, Filatyev et al. [22] presented ratios of global consumption speed and laminar burning velocity $\left(\mathrm{S}_{\mathrm{T}, \mathrm{GC}} / \mathrm{S}_{\mathrm{LO}}\right)$ of up 3.8 and Kobayashi et al. [23] presented data with $\mathrm{S}_{\mathrm{T}, \mathrm{GC}} / \mathrm{S}_{\mathrm{LO}}$ ratios of up to 8. Both of these results represent global consumption rates, which take into account flame surface density, averaged across the entire flame surface; a fact that suggests local instantaneous values could be considerably higher. On a local basis, Shepherd et al. [24] measured values of $\mathrm{S}_{\mathrm{T}, \mathrm{LC}} / \mathrm{S}_{\mathrm{LO}}$ up to 2.6 in equivalence ratios of 0.95 . Given that displacement velocities have been shown to be 2-3 times greater than consumption velocities [9], the local values of $\mathrm{S}_{\mathrm{T}, \mathrm{LD}} / \mathrm{S}_{\mathrm{LO}}$ presented in this work are not inconsistent with these other findings. Another explanation for the high values 
given by this technique is the advection process itself. The velocity measurement used for the advection are taken from particle motions directly in front of the preheat zone, around $0.5-1 \mathrm{~mm}$ in front of the inner reaction zone [16]. It could be that the advection velocities used to not fully reflect the translation of the inner reaction zone. However, given the complications of measurement process it has not been possible to make this assessment.

Assuming the high measurements of $\mathrm{S}_{\mathrm{T}, \mathrm{LD}}$ produced by the convection technique are truly representative of the flames burning velocity, the question of the mechanism by which this happens needs to be addressed. Possible mechanisms reported in literature include flame stretch and flame curvature which have been reported to effect local flame speeds due to their impact on the thermodiffusive transport through the flame. However, disagreement in literature exists to the role and dominance of these different mechanisms [7]. In the study of premixed stoichiometric methane-air combustion a notable study is that produced by Gran et al. [4]. In this work a 2-dimensional propagating flame was investigated using DNS. The results reported showed that flame curvature significantly effected the rate of flame displacement; in regions of negative curvature the displacement rate was enhanced while in regions of highly positive curvature negative flame speeds were produced. This response was linked to the diffusion of hydrogen produced in the flame. Gran et al. state that the consumption of methane primarily occurs because of the reaction $\mathrm{CH}_{4}+\mathrm{OH}(\mathrm{H})$ $\rightleftharpoons \mathrm{CH}_{3}+\mathrm{H}_{2} \mathrm{O}\left(\mathrm{H}_{2}\right)$ and that, since methane is abundant, it is the concentration of $\mathrm{H}$ and $\mathrm{OH}$ that determines the forward reaction rate of the flame. With a Lewis number of $0.18, \mathrm{H}$ is extremely mobile [4] making it more prone to the focusing or defocusing effects of curvature. In positive curvature, there is an enrichment of hydrogen in the burned region behind the flame front, due to the focusing effect, while at the same time a deficiency of hydrogen occurs near the unburned side. This process can significantly slow forward propagation and in cases of high positive curvature produce negative burning velocities. This mechanism could explain some of the data produced at the flame tip where high positive curvature occurs. With negative curvature, an enrichment of hydrogen, diffusing from the oxidation layer of the flame, occurs near the leading edge of the propagating flame enhancing the forward propagation rate. It is clear from these findings that any flow or flame profile aspects which aid the diffusion and concentration of hydrogen towards the preheat zone of the flame will result in an enhancement to its forward propagation rate.

\subsection{Fluid dynamic and flame curvature effects}

As stated in section 4.3, two of the mechanisms most discussed in literature that effect flame propagation are stretch and curvature. This section looks at these aspects in relation to the measure flame displacement speeds. Due to the uncertainty involved in the measurements around the flame tip, these regions have been removed from the comparisons.

For clarity, analysis of the first four time steps is presented, i.e. before the flame starts to interact with the vortex core; a region that has a lower pressure than the surrounding charge due to its rapid rotation. This lower pressure results in fewer reactant molecules present per unit volume, which in turn affects the apparent burning velocity. The additional influence of pressure would confuse any 
link between flame propagation and local flow structure investigated, therefore only the initial stages of the interaction are considered.

Flame stretch $(\mathrm{K})$ is composed of two terms: The first term is the fluid dynamic straining of the flame front due to non-uniformity in the flow field; a term that can be obtained by calculating the divergence of the flow velocity tangential to the flame front. The second term is slightly more complex and represents the stretch effect brought about by a curved flame front propagating or being convected in a direction normal to the flame surface. Combining these two terms, the flame stretch equation becomes [25]:

$$
K=\nabla \cdot \mathbf{V}_{s, t}+\left(\mathbf{V}_{f} \cdot \mathbf{n}\right)(\nabla \cdot \mathbf{n})
$$

Where $\mathbf{V}_{\mathrm{s}, \mathrm{t}}$ is the tangential component of the flow velocity, $\mathbf{V}_{\mathrm{f}}$ is the total flame velocity combining both advection and propagation elements and $\mathbf{n}$ is the unit vector normal to the flame surface.

For the results presented, the APIV data has been used to calculate the flame stretch in the two components through the planar sheet intersecting the centre line of the orifice (axial and radial components). The third component (circumferential) is included in the analysis by assuming rotational symmetry of the results about the orifice axis. The levels of stretch measured coincide with the values calculated for a counter rotating vortex pair by Hasegawa et al. [26]

The variation in burning velocity with flame stretch, is shown in Figure 13. From this data no significant correlation can be discerned. These findings appear to conflict with the work presented by Gu et al. [1] and Filatyev et al. [22], who have suggested that such a correlation exists based on globally averaged measurements. Taking such a global approach to the measurements presented here would relate an overall positive stretch rate with an average burning velocity above that the unperturbed laminar value. However, for the flame-flow interactions presented here, it is clear that, on a local basis there is no significant relationship of this nature and that flame stretch is not the primary cause of burning velocity enhancement.

Fig 13. Local burning velocity as a function of flame stretch

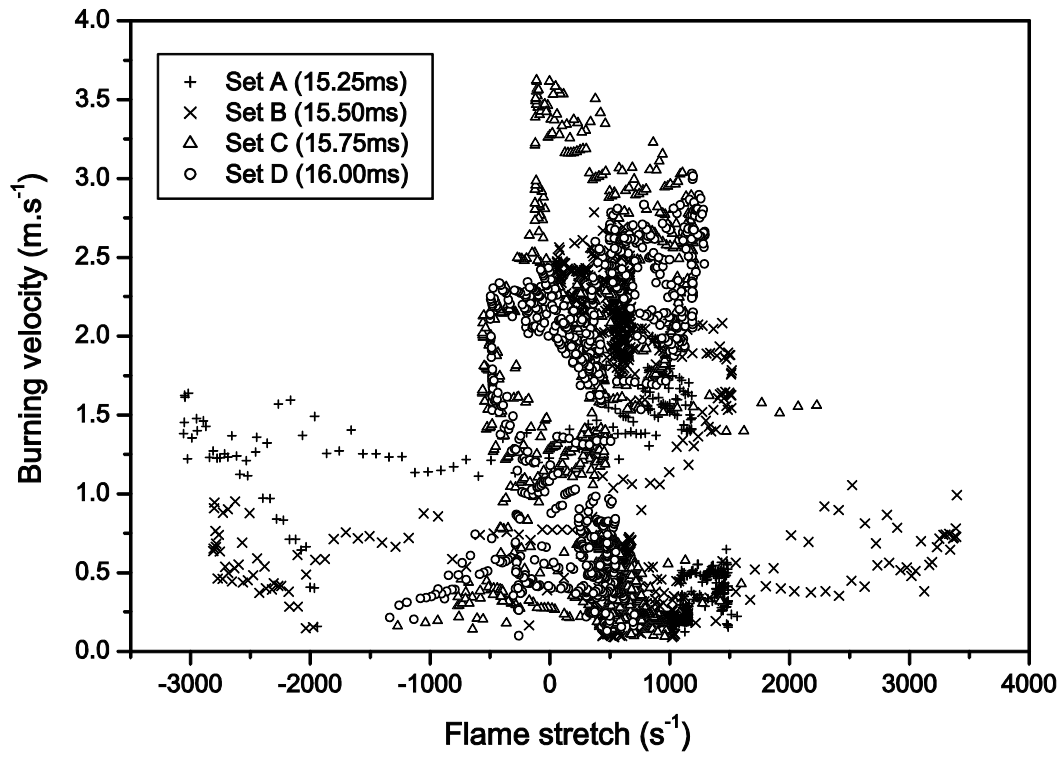

As presented by the work of Gran et al. [4] and Choi and Puri [5], it is clear that flame curvature could independently influence burning velocity due to the focusing/dispersion effects of the heat and mass from the flame. Figure 14 shows 
this relationship, plotting the inverse radius of curvature against burning velocity; positive curvature is defined as the flame being convex towards the unburned reactants.

Fig 14. Local burning velocity as a function of flame curvature

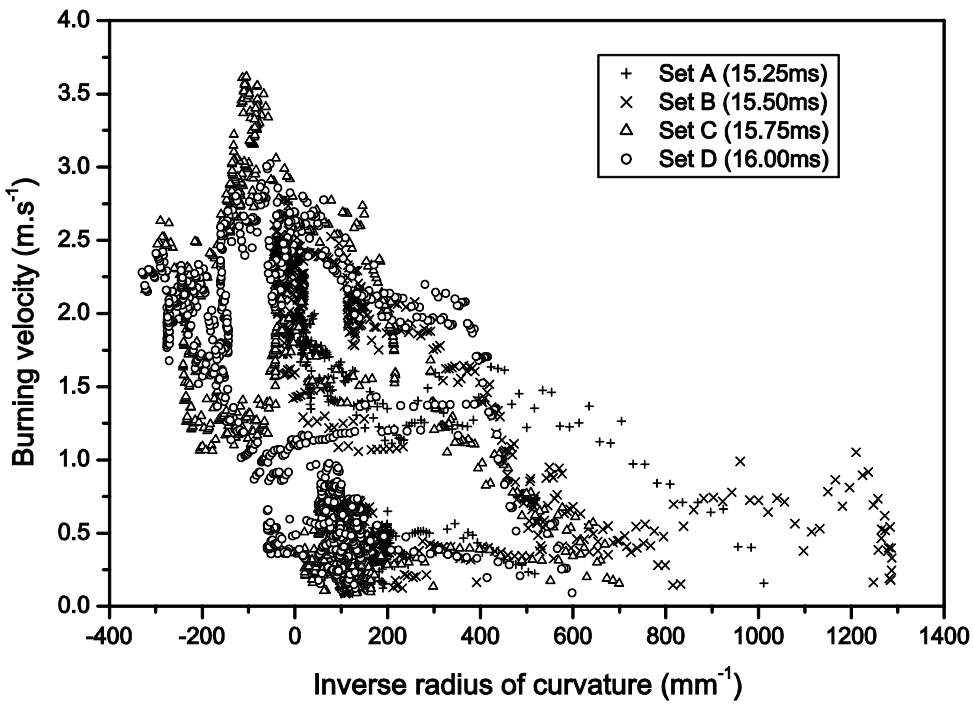

This figure indicates that there is some correlation between curvature and propagation rate, with regions of negative curvature exhibiting the highest burning velocities. This relationship generally supports the direct numerical simulation work carried out by Gran et al.[4]. Despite the discernable correlation between curvature and burning velocity observed, there is sufficient spread in the results to suggest that curvature is not the only influencing factor.

There are extended regions of zero or positive curvature which also contain enhanced rates of flame propagation. In examining the data in section 4.1, it was noted that the regions of high burning velocity consistently coincide with areas where the reactant flow runs nearly parallel with the flame surface. This relationship is quantified in Figure 15, where the angle of flow incidence is compared with local burning velocity. The angle of incidence is defined as 0 radians where the flow is normal to the flame surface and $\pi / 2$ radians where the flow is tangential to the flame.

Fig 15. Local burning velocity as a function of flow direction

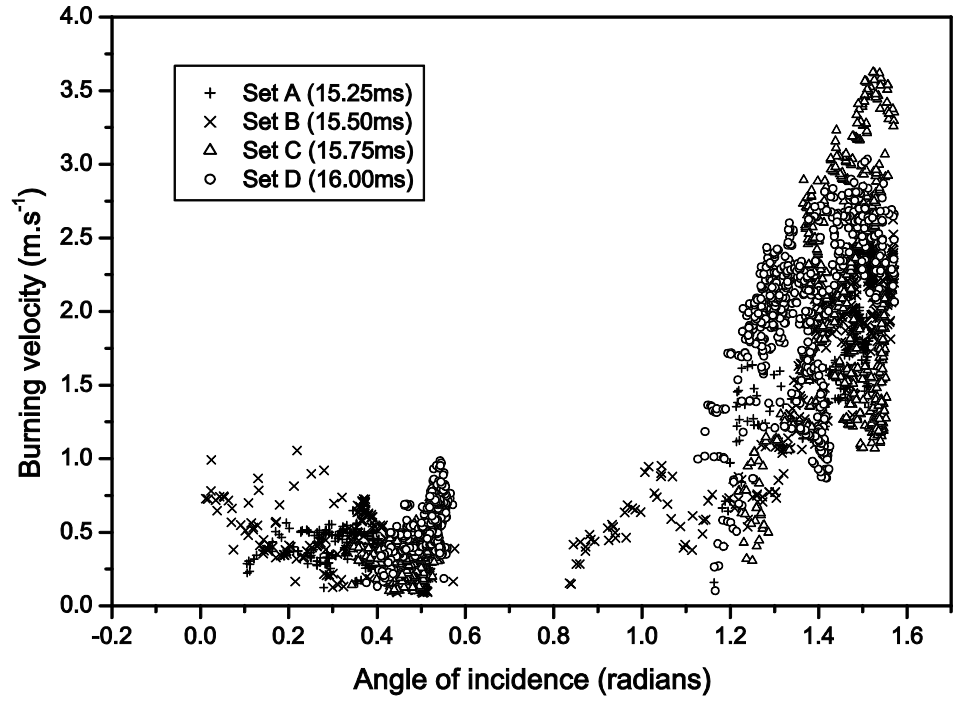


This figure demonstrates a surprising correlation between flow direction and burning velocity, with flows that are running close to parallel with the flame surface coinciding with high flame propagation rates. The reason for this link could be similar to the driving factors behind the effect of flame curvature. In the case of negative curvature, it is suggested that it is the focusing of hydrogen diffusion towards the unburned side of the flame front; the higher the negative curvature, the greater this focusing effect, enhancing the reaction. With angle of incidence, a flow dominated process drives the interaction, with fluid elements travelling tangentially through the preheat zone and the beginning of the inner reaction zone for longer. This process could mean that there is greater opportunity to receive both heat and hydrogen enrichment from the oxidation layer. By comparing both curvature and angle of incidence to burning velocity (figure 16) it is shown that, for these results, a strong correlation exists between burning velocity and regions that contain both negative curvature and highly tangential flows.

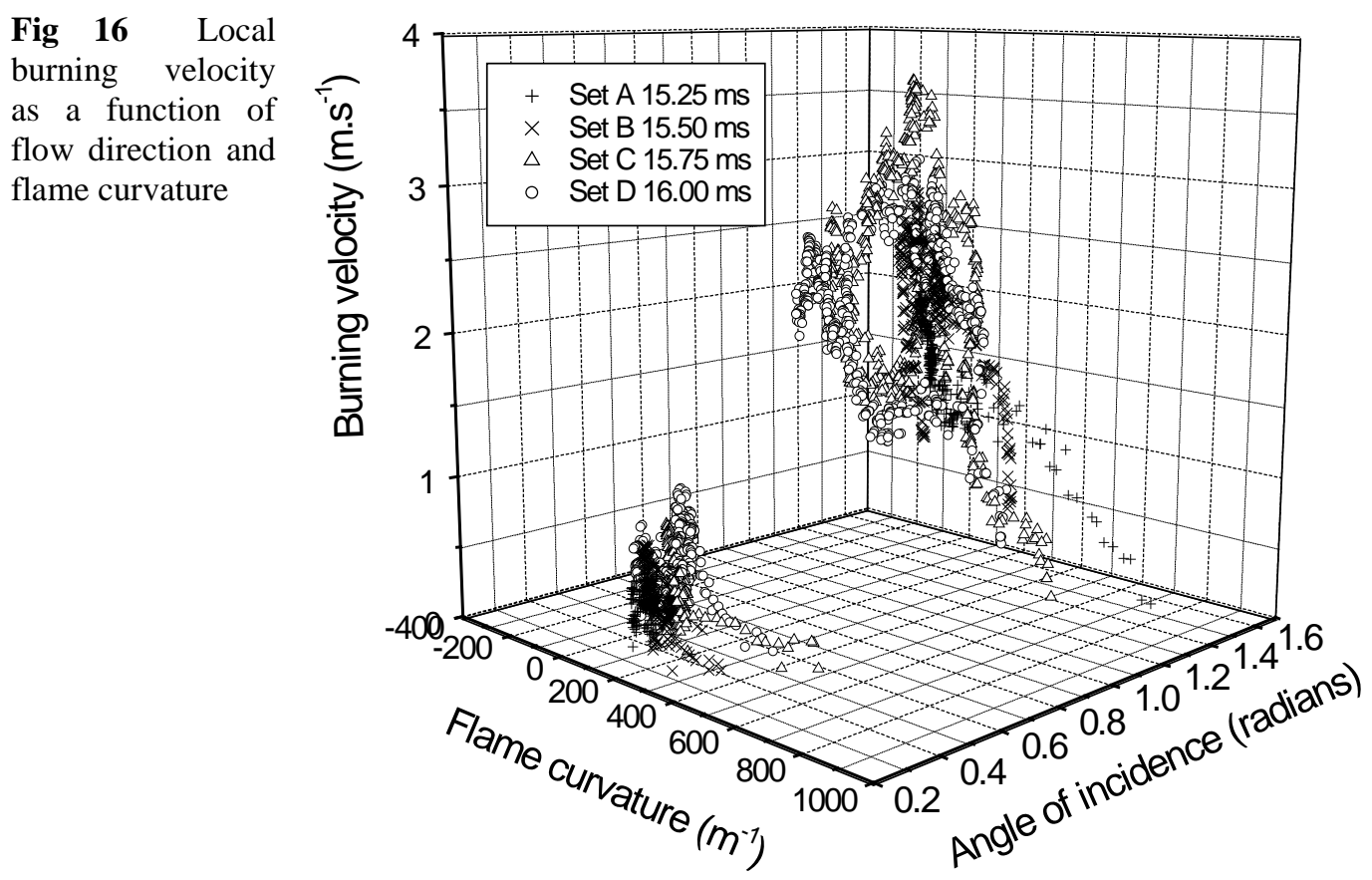




\section{Conclusion}

In this work a technique for the measurement of local burning velocity has been developed for the study of flame fronts interacting with flows containing high rotation. It has been shown that such rotational flows present a significant challenge for the measurement of burning velocity due to the high flow velocities tangential to the flame surface. The developed technique has subsequently been applied to the measurement of a single toroidal vortex interacting with a flame front propagating through a premixed charge of stoichiometric methane and air. This interaction produces a perturbed laminar flame that is both controllable and highly repeatable. Through this application, it has become apparent that a spatial offset exists between the location at which the flow velocity is measured, close to the preheat zone of the flame, and the inner reaction zone. This offset is brought about by the evaporation of the seeding particles used in the measurement process. Although small, typically between 0.5 and $1 \mathrm{~mm}$, such an offset could result in an incorrect convection velocity and could therefore effect the final burning velocity measurement.

The burning velocity data produced from the application of the proposed technique has demonstrated a significant enhancement to the flame propagation rate where the flame directly interacts with the rotating vortex; a finding that is agreement with a qualitative assessment of the flame progression. Away from this interaction with the main vortex core, the flame exhibits propagation rates similar to those published for unperturbed laminar combustion [18-21]. Although the increases in burning velocity measured using the described technique are high, in places up to 8 times the unperturbed laminar velocity, it has been shown that these values are not inconsistent with findings taken from the turbulent combustion of stoichiometric methane and air [22-24]. Since a substantial role is played by thin laminar zones in turbulent combustion [7] such a comparison can be made.

Analysis of the results produced has demonstrated little correlation between flame stretch and burning velocity when examined on a local basis, however strong correlations for both flame curvature and the angle of flow incidence have been identified. Based on the work by Gran et al. [4], it is suggested that these two effects both derive their influence from the processes through which hydrogen, produced by the dominant reaction of $\mathrm{CH}_{4}+\mathrm{OH}(\mathrm{H}) \rightleftharpoons \mathrm{CH}_{3}+\mathrm{H}_{2} \mathrm{O}\left(\mathrm{H}_{2}\right)$, is concentrated towards the unburned charge ahead of the flame front.

\section{References}

1 Gu, X.J., Haq, M.Z., Lawes, M., and Woolley, R., Laminar Burning Velocity and Markstein Lengths of Methane-Air Mixtures. Combust. Flame, 2000. 121: 41-58.

2 Chen, J.H. and Im, H.G. Correlation of Flame Speed with Stretch in Turbulent Premixed Methane/Air Flames. in Twenty-Seventh (International) Symposium on Combustion.: The Combustion Institute. 1998 pp 819-826

3 Echekki, T. and Chen, J.H., Analysis of the contribution of Curvature to Premixed Flame Propagation. Combust. and Flame, 1999. 118: 308-311.

4 Gran, I.R., Echekki, T. and Chen, J.H., Negative Flame Speed in an Unsteady Premixed Flame: A Computational Study in Twenty-Sixth (International) Symposium on Combustion. The Combustion Institute. 1996 pp 323-329

5 Choi, C.W. and Puri, I., Response of flame speed to positively and negatively curved premixed flames. Combust theory model, 2003. 7:203-220. 
6 Baum, M., Poinsot, T., Haworth, D.C., and Darabiha, N., Direct numerical simulation of H2/02/N2 flameswith complex chemistry in two-dimensional turbulent flows. J. Fluid Mech, 1994. 281: $1-32$.

7 Lipatnikov, A.N. and Chomiak, J., Molecular transport effects on turbulent flame propagation and structure. Prog Energ Combust, 2005. 31:1-71.

8 Driscoll, J.F., Turbulent premixed combustion: Flamelet structure and its effect of turbulent burning velocities Prog Energ Combust, 2008, 34:91-134

9 Shepherd, I.G., and Cheng, R.K., The Burning Rate of Premixed Flames in Moderate and Intense Turbulence combust flame, 2001, 127:2066-2075

10 Lipatnikov, A.N. and Chomiak, J., Turbulent Burning Velocity and Speed of Developing, Curved, and Strained Flames, Proc. Combust. Inst, 2002, 29:2113-2121

11 Renou, B., Boukhalfa, A., Puechberty, D., and Trinite, M., Local Scalar Flame Properties of Freely Propagating Premixed Turbulent Flames at Various Lewis Numbers. Combust. Flame, 2000. 123(4): 507-521.

12 Sinibaldi, J.O., Driscoll, J.F., Mueller, C.J., Donbar, J.M., and Carter, C.D., Propagation Speeds and Stretch Rates Measured Along Wrinkled Flames to Assess the Theory of Flame Stretch. Combust. Flame, 2003, 133:323-334.

13 E. J. Long E.J., Rimmer, J.E.T., Justham, J., Garner C.P., Hargrave, G.K., Richardson, D., and Wallace, S., The Influence of In-Cylinder Turbulence upon Engine Performance within a Direct Injection IC Engine, in The seventh international Conference on Modelling and Diagnostics for Advanced Engine Systems (COMODIA) JSME 08-202, 2008 Sapporo

14 Hartung, G. Hult, J., Balachandran, R., Mackley, M.R. and Kaminski, C.F., Flame front tracking in turbulent lean premixed flames using stereo PIV and time-sequenced planar LIF of OH, Appl.Phys.B, Lasers Opt., 96:843-862

15. Jarvis, S. and Hargrave, G.K., Premixed Flame Propagation and Interaction with Turbulent Flow Structures in a Semi-Confined Combustion Chamber, in Wolfson School of Mechanical and Manufacturing Engineering. 2003, Loughborough University: Loughborough.

16 Heeger, C., Bohm, B., Ahmed, S.F., Gordon, R., Boxx, I., Meier, W., Dreizler, A., and Mastorakos, E., Statistics of relative and absolute velocities of turbulent non-premixed edge flames following spark ignition, Proc. Combust. Inst. 2009 32:2957-2964

17 Anandarajah, K., Hargrave, G.K., and Halliwell, N.A. Time-Resolved Digital Particle Image Velocimetry: Error Reduction from Normalisation by Signal Strength. in International Conference on Advanced Optical Diagnostics in Fluids, Solids, and Combustion. 2004. Tokyo.

18 Tahtouh, T., Halter, F., and Mounaim-Rousselle, C., Measurement of laminar burning speeds and Markstein lengths using a novel methodology, Combust. Flame, 2009, 159: 1735-1743

19 Hassan, M.I., Aung, K.T., Faeth, G.M., Measured and predicted properties of laminar premixed methane/air flames at various pressures, Combust. Flame 1998 115:539- 550.

20 Halter, F., Chauveau, C., Djebai"li-Chaumeix, N., and Gokalp, I., Characterization of the effects of pressure and hydrogen concentration on laminar burning velocities of methanehydrogen- air mixtures, Proc. Combust. Inst., 2005, 30:201- 208.

21 Coppens, F.H.V., De Ruyck, J., and Konnov, A.A., Effects of hydrogen enrichment on adiabatic burning velocity and NO formation in methane + air flames, Exp. Therm. Fluid Sci. 2007, 31:437- 444.

22 Filatyev, S.A., Driscoll, J.F., Carter, C.D., and Donbar, J.M., Measured Properties of Turbulent Premixed Flames for Model Assessment, Including Buning Velocities, Stretch Rates, and Surface Densities. Combust. Flame, 2005. 141:1-21.

23 Kobayashi, H, Kawahata, T., Seyama, K., Fujimari T., and Kim J.,Relationship between the smallest scale of flame wrinkles and turbulence characteristics of high-pressure hightemperature turbulent premixed flames Pro. Combust. Inst., 2002, 29:1793-1800

24 Shepherd, I.G., Bourguignon, E., Michou, Y., and Gokalp, I., The burning rate in turbulent Bunsen flames, in The Twenty-Seventh Symposium (International) on Combustion The Combustion Institute, 1998, 909-916

25 Chung, S.H. and Law, C.K., An invariant derivation of flame stretch. Combust. Flame, 1984. 55:123-125.

26 Hasegawa, T., Morooka, T., and Nishiki., Mechanism of Interaction between a vortex pair and a premixed flame. Combust. Sci. Tech., 2000, 150:115-142 